\title{
Mécanisme d'absorption intestinale des acides gras à longue chaîne : rôle émergent du CD36
}

\author{
Thi Thu Trang TRAN \\ Marjorie BUTTET \\ Véronique TRAYNARD \\ Philippe BESNARD \\ Hélène POIRIER \\ Isabelle NIOT \\ Physiologie de la nutrition et toxicologie
(Nutox),
UMR U866 Inserm/université de
Bourgogne/AgroSup Dijon
1 Esplanade Erasme,
21000 Dijon, France
$<$ isabelle.niot@u-bourgogne.fr >
}

Article reçu le $1^{\text {er }}$ juin 2012

Accepté le 8 juin 2012

\begin{abstract}
Mechanism of Intestinal Absorption of Long chain Fatty acid: Emerging role of $\mathrm{CD} 36$

Excessive lipid intake, associated with a qualitative imbalance, favors the development of obesity and associated diseases. From organs involved in the lipid homeostasis, the small intestine remains the most poorly known although it is responsible for the lipid bioavailability and largely contributes to the regulation of postprandial hypertriglyceridemia. The mechanism of long chain fatty acid (LCFA) intestinal absorption is not totally elucidated. Over the two last decades, cloning of lipid binding proteins (LBP), proteins involved in trafficking and metabolic fate of LCFA in gut have provided new insights on cellular and molecular mechanisms involved in fat absorption. The synthesis of recent literature indicates that intestine is able to adapt its absorption capacity to the fat content of the diet. This adaptation takes place through a fat-coordinated induction of LBP and apolipoproteins. CD36 could operate as a lipid sensor responsible for a transducing signal related to the lipid content of the diet at the origin of this intestinal adaptation. This lipid-mediated metabolic response may lead to the formation of large chylomicrons rapidly degraded in the blood. All together, these new data indicate that this intestinal lipid sensing mechanism may be a therapeutic target for reducing the postprandial hypertriglyceridemia and associated cardiovascular risks.
\end{abstract}

Key words: intestine, CD36, lipid-binding proteins, intestinal adaptation, sensing, lipid absorption, postprandial hypertriglyceridemia

cipe à la régulation de la prise alimentaire en particulier par l'intermédiaire de la sécrétion $d^{\prime}$ hormones intestinales (CCK, GLP-1, GLP2...GIP) (Steinert et Beglinger, 2011) et de l'apolipoprotéine AIV qui est constitutive des lipoprotéines intestinales (Tso et Liu, 2004). L'ensemble de ces fonctions fait de l'intestin un organe pertinent dans l'étude de la mise en place de l'obésité et des maladies associées. Plus particulièrement, les perturbations du métabolisme lipidique intestinal peuvent retentir sur les capacités d'absorption et sur la lipémie postprandiale et affecter plus largement le métabolisme des autres lipoprotéines (Mora et al., 2008).

En ce qui concerne l'absorption intestinale des lipides, les mécanismes cellulaires et moléculaires ne sont pas encore totalement élucidés. Cela s'explique par le fait que l'intestin a longtemps été considéré comme une simple barrière passive capable d'absorber de grandes quantités de lipides alimentaires. Étant donné les rôles fondamentaux des acides gras à longue chaîne (AGLC) dans la cellule (fonctionnel, énergétique, métabolique et de signalisation cellulaire), on conçoit que leur biodisponibilité ne soit pas limitante. Le fait que l'excrétion fécale des lipides soit de l'ordre de $5 \%$ chez l'homme malgré de grandes variations de l'apport lipidique et que la stéatorrhée soit diagnostiquée dès que la perte excède $7 \%$ souligne l'efficacité de I'absorption intestinale des AGLC (Ross, 1993). Ces dernières années plusieurs lipid-binding proteins (LBP) ont été identifiées au niveau de l'entérocyte montrant la complexité du mécanisme d'absorption et suggérant que des modifications de leur niveau d'expression intestinale

Pour citer cet article : Tran TTT., Buttet M, Traynard V, Besnard P, Poirier H, Niot I. Mécanisme d'absorption intestinale des acides gras à longue chaîne : rôle émergent du CD36. OCL 2012; 19(4) : 200-208. doi : 10.1684/ocl.2012.0461 
retentissent sur l'efficacité de l'absorption et la lipémie postprandiale, et plus généralement sur I'homéostasie des lipides de l'organisme (Niot et al., 2009). $C^{\prime}$ est pourquoi, cette revue abordera le rôle des LBP en particulier les membranaires dans le mécanisme d'absorption des lipides et sa régulation.

\section{Absorption intestinale des lipides alimentaires}

L'alimentation apporte de 30 à $40 \%$ de l'énergie sous forme de lipides, principalement des triglycérides (TG). Du fait de leur caractère hydrophobe, I'absorption des TG au niveau intestinal est un processus complexe comportant plusieurs étapes (Niot et al., 2009) (figure 1): le captage des AGLC, le trafic intracellulaire, la synthèse et la sécrétion des chylomicrons.

À l'arrivée dans la lumière intestinale, les lipides alimentaires, principalement des TG sont hydrolysés en monoglycéride (MG) et AGLC grâce aux lipases gas-

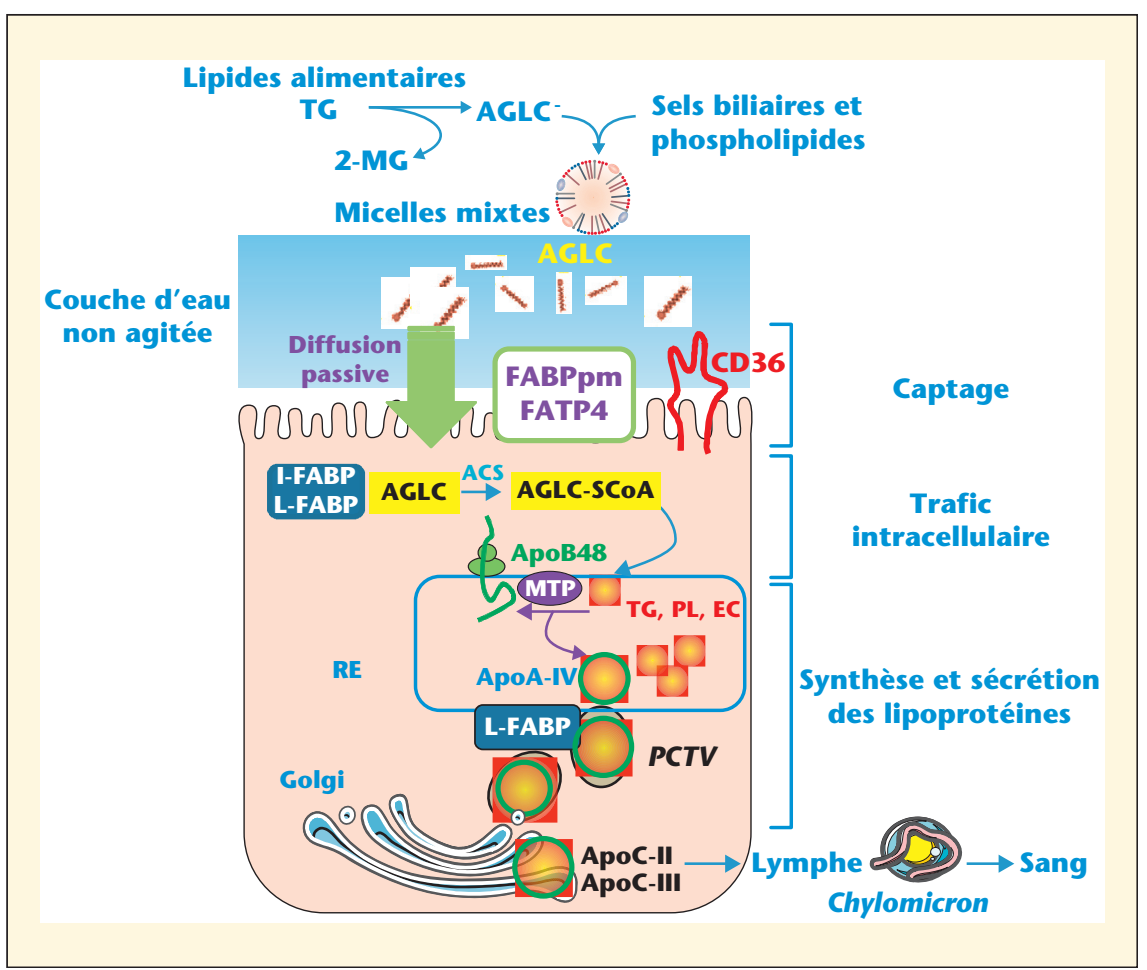

Figure 1. Principales étapes de l'absorption intestinale des AGLC.

AGLC : Acide gras à longue chaîne ; MG : monoglycérides; TG : triglycérides; PL : phospholipides; EC: ester de cholestérol; LBPs: lipid binding protein; CD36: fatty acid transporter; FATP4 : fatty acid transporter protein $4 ;$ I-FABP : intestinal fatty acid-binding protein ; L-FABP : liver fatty acid-binding protein; ACS: Acy COA synthétase; AGLC-SCOA: Acyl COA; ACBP : Acyl CoA-binding protein; MTP: microsomal triglyceride transfer protein ; ApoB48: apolipoprotéine $B 48$; ApoA-IV : apolipoprotéine A-IV ; RE : réticulum endoplasmique. fait de la présence de pompes à protons au niveau de la membrane apicale (figure 1). Ce microenvironnement est à l'origine de la dissociation micellaire qui libère les AGLC protonés à proximité immédiate de la surface de la bordure en brosse des entérocytes (Shiau et al., 1985) et facilite leur captage par diffusion passive (Niot et al., 2009). Ce constat pose la question du rôle physiologique des LBP membranaires identifiées dans le processus d'absorption des AGLC: la FABPm, le FATP4 et CD36.

\section{La FABPpm}

La FABPpm est exprimée aussi bien dans la bordure en brosse que dans les membranes cellulaires latérales des entérocytes en cours de différenciation et différenciés (Stremmel et al., 1985b). Le fait que l'utilisation de l'anticorps antiFABPpm sur des explants jéjunaux réduise le captage des acides gras montre qu'elle pourrait jouer un rôle dans cette étape membranaire (Stremmel, 1988). De plus, la FABPpm est aussi présente dans la mitochondrie puisqu'elle est I'homologue de la mitochondrial aspartate-aminotransferase (mASpAT) qui catalyse les réactions de transamination reliant le cycle de l'urée au cycle de Krebs (Stump et al., 1993). Ces données montrent que des études plus approfondies sont nécessaires pour déterminer si la FABPpm a un rôle physiologique dans I'absorption intestinale des lipides. L'invalidation de ce gène n'a toujours pas été réalisée à ce jour.

\section{La FATP4}

La FATP4 est une protéine membranaire appartenant à la famille des FATP qui comporte cing FATP murines et six FATP humaines et dont chaque membre a une expression tissulaire spécifique (Doege et Stahl, 2006). Au niveau intestinal, la FATP4 est majoritairement exprimée au niveau des entérocytes hautement différenciés du jéjunum. Sa localisation entérocytaire fait l'objet de discussions puisqu'elle a été initialement décrite au niveau des membranes microvillositaires (Stahl et al., 1999), mais serait plus majoritairement présente dans le réticulum endoplasmique (RE) (GarciaMartinez et al., 2005; Milger et al., 2006). II a été montré en utilisant des entérocytes isolés de souris dépourvus de couche d'eau non agitée, qu'il existe une corrélation entre l'efficacité de captage des AGLC et l'expression de la FATP4 
(Gimeno et al., 2003 ; Stahl et al., 1999). Ces données indiquent que la FATP4 participe au captage des AGLC au niveau entérocytaire. Cependant, la structure de la protéine FATP4 essentiellement cytosolique, avec une courte séquence extracellulaire du côté $\mathrm{N}$-terminal qui ne contient pas de domaine de liaison aux acides gras (Gertow et al., 2004 ; Stahl et al., 2001), met en doute son rôle de transporteur des AGLC. En revanche, il est maintenant démontré que la FATP4 porte une activité Acyl-CoA synthétase des acides gras à longues et très longues chaînes (Hall et al., 2005). Ainsi, la FATP4 en activant les AGLC en AcylCoA participe au maintien d'un gradient favorable à leur entrée dans l'entérocyte par diffusion passive (Milger et al., 2006). L'ensemble de ces données explique pourquoi la délétion de FATP4 au niveau intestinal chez la souris ne modifie pas l'efficacité d'absorption intestinale des lipides alimentaires (captage et excrétion fécale). On observe cependant une accumulation légère de TG au niveau des entérocytes quand ces souris déficientes en FATP4 sont nourries avec un régime hyperlipidique (Shim et al., 2009). Ce résultat souligne son rôle dans le métabolisme entérocytaire des TG qui s'effectue principalement au niveau du RE (Milger et al., 2006). Le fait que la diminution d'expression de la FATP4 (Frochot et al., 2012) et que le polymorphisme Gly-209-Ser sur le gène de la FATP4 observé chez l'homme se traduisent par une réduction de l'hypertriglycéridémie (Gertow et al., 2004) est en accord avec cette hypothèse.

\section{$C D 36$}

Le CD36 est une glycoprotéine multifonctionnelle capable de lier les AGLC saturés ou non, ionisés, avec une affinité de I'ordre du nanomolaire (Ibrahimi et al., 1996) mais également le collagène, les phospholipides anioniques, les LDL oxydées, le Plasmodium falciparum, la thrombospondine et la $\beta$-amyloïde (Martin et al., 2011 ; Silverstein et Febbraio, 2009 ; Su et Abumrad, 2009). Le CD36 est localisé principalement au niveau des membranes des bordures en brosse des deux tiers supérieurs des villosités dans la partie proximale de l'intestin grêle (Lobo et al., 2001 ; Poirier et al., 1996). L'expression intestinale du CD36 est étroitement corrélée avec la teneur en lipides du régime. Alors qu'un régime pauvre en lipides diminue son expression (Sukhotnik et al., 2001), un régime hyperlipidique chronique l'induit fortement (Poirier et al., 1996). Cependant, à la différence de l'adipocyte et du myocyte, la contribution significative de CD36 dans le captage entérocytaire des AGLC est mise en doute. En effet, selon les auteurs, la délétion du gène du CD36 diminue le captage des AGLC par les entérocytes isolés dépourvus de couche d'eau non agitée (Nassir et al., 2007), ou n'a pas d'effet (Drover et al., 2005). Nos travaux ont démontré la pertinence physiologique de ces dernières données puisque des anses jéjunales isolées in situ de souris sauvages et déficientes en CD36 qui conservent la couche d'eau non agitée et la dynamique de l'absorption, ont la même efficacité de captage (Tran et al., 2011). Ces derniers résultats expliquent pourquoi l'invalidation du gène de CD36 chez la souris ne se traduit pas in vivo par une modification de l'absorption nette des AGLC excepté pour des acides gras à très longues chaînes rares dans l'alimentation (Drover et al., 2008 ; Nassir et al., 2007 ; Nauli et al., 2006). Cependant, le CD36 participe bien au mécanisme d'absorption des lipides alimentaires puisque sa déficience réduit considérablement la sécrétion lymphatique des chylomicrons qui sont moins riches en TG et donc de plus petites tailles (Masuda et al., 2009 ; Nauli et al., 2006). Ces effets sont associés à une rétention de TG au niveau entérocytaire en régime hyperlipidique chronique (Drover et al., 2005). Ces données démontrent que le CD36 joue un rôle très important dans la formation des chylomicrons mais pas comme transporteur efficace des lipides.

En conclusion, l'existence de la couche d'eau non agitée en plus des propriétés structurelles et fonctionnelles des LBP membranaires indique que la contribution d'un transport facilité dans le captage intestinal des acides gras alimentaires n'est probablement pas prépondérante en période postprandiale. En revanche, la diffusion passive, système de transport à forte capacité mais à faible affinité, est particulièrement bien adaptée et éviterait ainsi que le captage des AGLC ne devienne un facteur limitant dans I'absorption intestinale des lipides après un repas (Chow et Hollander, 1979).

Il est important de noter que l'efficacité de cette diffusion passive est conditionnée par le maintien d'un gradient de concentration en AGLC entre les milieux extra- et intracellulaire dépendant de l'efficacité du métabolisme entérocytaire des AGLC. Cependant, pour des concentrations plus faibles, en situation interprandiale par exemple, la diffusion facilitée pourrait s'avérer plus efficace, du fait d'une plus forte affinité pour les AGLC.

\section{Trafic intracellulaire des acides gras à longue chaîne}

Une fois dans le cytoplasme, les AGLC sont pris en charge par des fatty acidbinding proteins (I et L-FABP) dont les fonctions respectives ne sont pas totalement élucidées. Cependant, leur liaison avec les AGLC en maintenant le gradient de concentration favorise leur captage par diffusion passive (Niot et al., 2009). Quand des AGLC sont délivrés au pôle apical des entérocytes, ils sont préférentiellement liés à la I-FABP, isoforme, uniquement exprimée dans cet organe, qui requiert un mécanisme collisionnel avec les phospholipides membranaires pour transférer les AGLC. Ces deux propriétés suggèrent que la IFABP est impliquée dans le transfert vectoriel des AGLC vers le RE, site de réestérification des acides gras (Corsico et al., 1998). Puisque, l'échange des AGLC pour la L-FABP s'effectue par diffusion aqueuse, elle serait plutôt à I'origine de la formation d'un pool intracellulaire d'AGLC. Par ailleurs, elle serait un partenaire des PPARs. Enfin au niveau intestinal, la L-FABP participe au transport intracellulaire des préchylomicrons comme nous le décrirons plus tard. En accord avec cette dernière hypothèse, I'invalidation de la L-FABP conduit chez la souris soumise à une charge en lipides à une moindre sécrétion de TG associée à leur rétention entérocytaire (Newberry et al., 2006).

\section{Synthèse et sécrétion \\ des chylomicrons}

Les AGLC sont ensuite activés en AcylCoA par des Acyl-CoA synthétases (ACS). Pendant la période postprandiale, les Acyl-CoA formés sont ré-estérifiés préférentiellement en TG avant d'être transloqués dans le RE avec les phospholipides et les esters de cholestérol par la microsomal triglyceride transfer protein (MTP). Ce transfert conduit à la lipidation d'une apolipoprotéine B48 (ApoB48), forme tronquée de l'ApoB100, spéci- 
fique de l'intestin au moins chez I'homme et le hamster. L'assemblage des préchylomicrons nécessite plusieurs étapes (Iqbal et Hussain, 2009). En effet, I'association d'une ApoB48 avec une faible quantité de lipides (TG et phospholipides) par la MTP constitue une lipoprotéine primordiale qui fusionne avec des gouttelettes lipidiques contenant des TG et des esters de cholestérol, formée indépendamment dans le RE par la MTP. La MTP est indispensable àficience chez I'homme et la souris (par mutation ou transgenèse) est à l'origine de l'abêtalipoprotéinémie qui se caractérise par une malabsorption sévère des lipides caractérisée par une stéathorrée, une rétention entérocytaire de TG et l'absence de sécrétion de lipoprotéines contenant I'ApoB48 (tableau 1) (Berriot-Varoqueaux et al., 2001 ; Xie et al., 2007). Cette étape constitue donc la première étape limitante de la formation des chylomicrons. L'ApoAIV est aussi fondamentale au niveau de cette étape puisqu'elle favoriserait la lipidation en stabilisant le préchylomicron et/ou I'ApoB48 (Mansbach et Gorelick, 2007 ; Weinberg et al., 2012).

Ces préchylomicrons sont ensuite transportés vers I'appareil de Golgi par des vésicules issues du RE appelées prechylomicrons transfert vesicles (les PCTV) qui du fait de leur grande taille, sont retrouvées uniquement au niveau intestinal. Les préchylomicrons subissent dans I'appareil de Golgi des étapes de maturation (glycosylation) et acquièrent différentes apolipoprotéines comme les apolipoprotéines CII, CIII et A1 (ApoCII, ApoCIII, ApoA1). Ce transfert constitue la seconde étape limitante de I'absorption des lipides. II requiert la L-FABP pour initier la génération des PCTV au niveau du RE (Neeli et al., 2007) ou d'autres protéines pour leur fusion avec les membranes de I'appareil de Golgi comme GTPase Sar $1 \mathrm{~b}$ ou vesicule associated membrane protein 7 (VAMP7). Les chylomicrons sont sécrétés dans la lymphe et rejoignent la circulation sanguine ou leurs TG sont dégradés par la lipoproteine lipase (LPL).

L'activité de la LPL est considérée comme le régulateur majeur de la lipémie. Or, en plus de son niveau d'expression, l'activité de la LPL est régulée par le rapport ApoCII/CIII porté par la lipoprotéine. En effet, I'ApoCII est un activateur puissant de la LPL alors que I'ApoC-III est un inhibiteur (Jong et al., 1999). Même si le niveau de ces apolipoprotéines peut être modulé par échange avec les lipoprotéines hépatiques dans le sang, le niveau acquis par les chylomicrons dans l'entérocyte conditionne également l'efficacité de la LPL (Olivecrona et Beisiegel, 1997). De plus, la clairance des chylomicrons dépend également du nombre et de la taille des lipoprotéines. En effet, I'activité de la LPL est plus efficace sur un petit nombre de gros chylomicrons que sur grand nombre de petits chylomicrons (Martins et al., 1996 ; Xiang et al., 1999). Ce dernier critère reflète finalement l'efficacité de la lipidation de I'ApoB48.

En conclusion, hormis la MTP, les LBP ne sont pas indispensables au mécanisme d'absorption (tableau 1). Cependant, elles semblent faciliter à différents niveaux, la synthèse et la sécrétion des chylomicrons, en particulier en situation de challenge lipidique. Ce qui explique pourquoi leur déficience n'altère pas l'absorption globale des lipides (altération des pertes fécales en lipides) mais plutôt la triglycéridémie postprandiale (Niot et al., 2009).

\section{Régulation de I'absorption intestinale par les lipides alimentaires}

La question qui se pose est de savoir si l'intestin possède de manière constitutive la capacité à absorber de grandes quantités de lipides ou bien s'il existe une réponse métabolique adaptative en fonction de la quantité des lipides présents dans le régime. Cette seconde possibilité suggère qu'une dérégulation de cette adaptation puisse se traduire par des pertes fécales en lipides et/ou des dyslipidémies postprandiales et des rétentions de TG entérocytaires. II est admis chez l'homme et la souris que l'augmentation de la teneur en lipides se traduit par une augmentation de la taille des chylomicrons plutôt que de leur nombre (Cartwright et Higgins, 1999 ; Karpe et al., 1997). Comme le montre le tableau 1, le fait que l'expression de plusieurs LBP (MTP, CD36, L-FABP) et apolipoprotéines (ApoIV) soit régulable in vitro par les AGLC laisse supposer que l'efficacité de la formation des chylomicrons puisse être modulée par les lipides alimentaires. Nos travaux ont confirmé cette hypothèse, puisqu'un segment intestinal isolé in situ de souris nourries avec un régime hyperlipidique chronique possède une plus grande capacité à absorber les AGLC (augmentation de la capacité de captage et du métabolisme lipidique sans rétention de TG entérocytaire) que celui de souris nourries avec un régime normolipidique (Petit et al., 2007). Ces modifications expliquent pourquoi in vivo, un régime hyperlipidique chronique ne conduit pas à l'augmentation des pertes fécales en lipides. Cette adaptation intestinale s'explique par deux phénomènes complémentaires: l'induction de la prolifération intestinale et l'induction coordonnée de l'expression des gènes impliqués dans la formation des chylomicrons (CD36, FATP4, l et LFABP, MTP, ApoAIV et ApoCII) (Petit et al., 2007). De plus, il est intéressant de noter que cette régulation est réversible et dépendante de la teneur en lipides du régime (de Wit et al., 2011 ; Kondo et al., 2006 ; Petit et al., 2007). En effet, en utilisant un régime contenant différentes quantités de lipides (10\%, $20 \%, 30 \%$ et $45 \%$ de lipides en masse), de Wit et al., 2011 ont montré que le nombre de gènes régulés et l'amplitude de leur induction sont corrélés à la quantité de lipides du régime.

On note que I'expression de I'ApoCIII entérocytaire est diminuée alors que celle de I'ApoCIl est induite en cas de régime hyperlipidique riche en AGLC insaturés (Kondo et al., 2006 ; Petit et al., 2007). Par conséquent, l'augmentation du rapport ApoCII/ApoCIII et de la taille des chylomicrons expliqueraient la plus faible hypertriglycéridémie postprandiale observée paradoxalement après ce type de régime hyperlipidique chez le rongueur (Petit et al., 2007) et retrouvée chez I'homme (Defoort et al., 2011). Cette adaptation intestinale est donc un facteur émergent de la régulation de la triglycéridémie postprandiale.

De manière intéressante, au moins chez la souris, l'adaptation intestinale à la teneur en lipides du régime contribue à la régulation de l'homéostasie lipidique (de Wit et al., 2011 ; Kondo et al., 2006) puisqu'un défaut est associé au développement de l'obésité (Kondo et al., 2006). Ces différents constats indiquent que la compréhension du mécanisme de l'adaptation du métabolisme entérocytaire à la teneur en lipides du régime devrait permettre d'envisager des actions thérapeutiques 


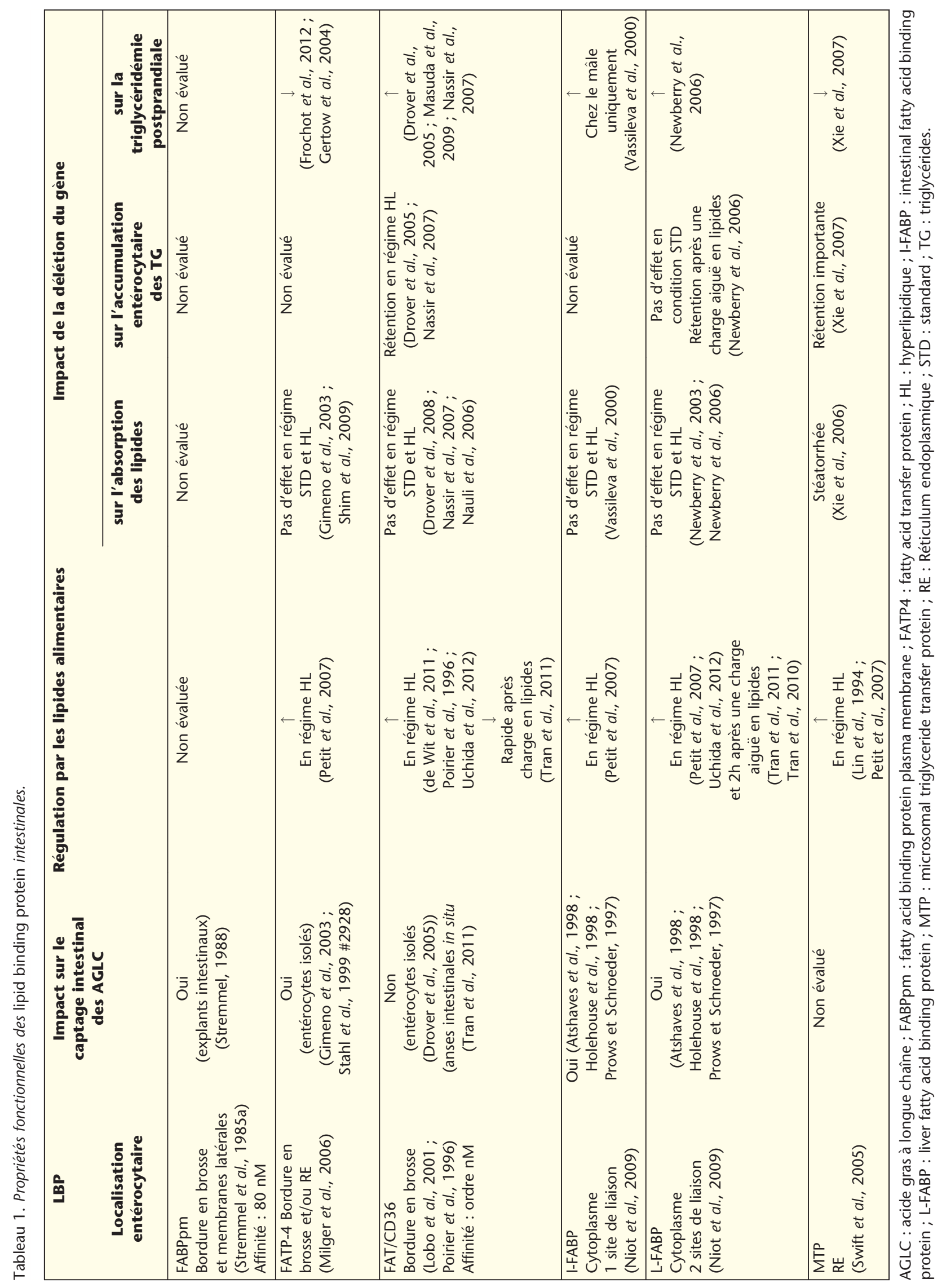

204 OCL VOL. 19 NN$^{\circ} 4$ juillet-août 2012 
pour limiter la triglycéridémie postprandiale mais également les maladies métaboliques associées.

Plus récemment, des études ont rapporté que I'adaptation génique observée en régime chronique s'exerçait également suite à une seule charge en lipides (Tran et al., 2011; Uchida et al., 2012). Cette donnée démontre que l'adaptation ne résulte pas des effets métaboliques secondaires au régime hyperlipidique chronique mais serait directement dépendante de la présence des lipides alimentaires dans la lumière intestinale. En effet, un gavage avec de I'huile riche en AGLC déclenche en moins de deux heures une induction du taux d'ARNm et de la protéine L-FABP et de la MTP (Tran et al., 2011 ; Tran et al., 2010 ; Uchida et al., 2012). Pour exister, cette adaptation suggère l'existence au niveau entérocytaire, d'un système de détection de la teneur en lipides du repas. Parmi les $L B P$, la régulation et les propriétés fonctionnelles font du CD36 un candidat pertinent.

\section{Détection entérocytaire des lipides alimentaires : rôle émergent du CD36}

Au contraire de la MTP et de la L-FABP, nous avons observé que la protéine CD36 intestinale était, quant à elle, diminuée une heure après un gavage avec de I'huile chez les rongeurs (Tran et al., 2011). Des études d'immunohistochimie ont démontré que la disparition partielle du CD36 des membranes microvillositaires (environ $75 \%$ ) s'exerce uniquement après un repas contenant des lipides (3\% en masse). Cette disparition membranaire s'explique par une dégradation par le protéasome, lipide-dépendante consécutive à la polyubiquitination du CD36 (Tran et al., 2011). Ce phénomène rapide constitue un argument supplémentaire montrant que le CD36 n'est pas un transporteur efficace des lipides pendant leur phase de digestion. En revanche, ce type de régulation est souvent observé pour les récepteurs associés à une signalisation qui correspond à la désensibilisation de la cellule vis-à-vis du ligand. Or, il a déjà été rapporté que la protéine CD36 peut également jouer un rôle de récepteur vis-à-vis de plusieurs de ses ligands (Martin et al., 2011). C'est le cas, au niveau des macrophages pour les LDL oxydés, au niveau des cellules endo- théliales pour les thrombospondines (Silverstein et Febbraio, 2009) et enfin au niveau des cellules réceptrices gustatives des bourgeons du goût pour les AGLC (El-Yassimi et al., 2008 ; Laugerette et al., 2005). Dans tous les cas, les signaux intracellulaires déclenchés par les ligands du CD36 impliquent le recrutement et I'activation des Src kinases et/ou des mitogen-activated protein kinases (MAPKs) (Silverstein et Febbraio, 2009).

En accord avec ces données, nos résultats obtenus ex vivo sur des segments intestinaux de souris ont démontré que les AGLC déclenchent après cinq minutes de contact, I'activation des extracellular signal regulated kinase $1 / 2$ (ERK $1 / 2$ ) suivie par une induction du taux d'ApoB48 (après dix minutes) et de la MTP (après vingt minutes). On retrouve après vingt minutes la dégradation du CD36 concomitante avec une diminution du niveau de phosphorylation des ERK $1 / 2$ correspondant sans doute au phénomène de désensibilisation (Tran et al., 2011). Cette régulation est dépendante du CD36 puisqu'elle est absente dans les segments intestinaux provenant d'animaux déficients en CD36 (Tran et al., 2011). La phosphorylation des ERK1/2 a déjà été rapportée comme étant impliquée dans la formation et la sécrétion des lipoprotéines hépatiques et intestinales (Federico et al., 2006 ; Tsai et al., 2007). Ces régulations ex vivo ont une pertinence physiologique puisque chez les souris déficientes en CD36, I'induction des protéines MTP et L-FABP observée deux heures après un gavage est abolie (données non présentées).

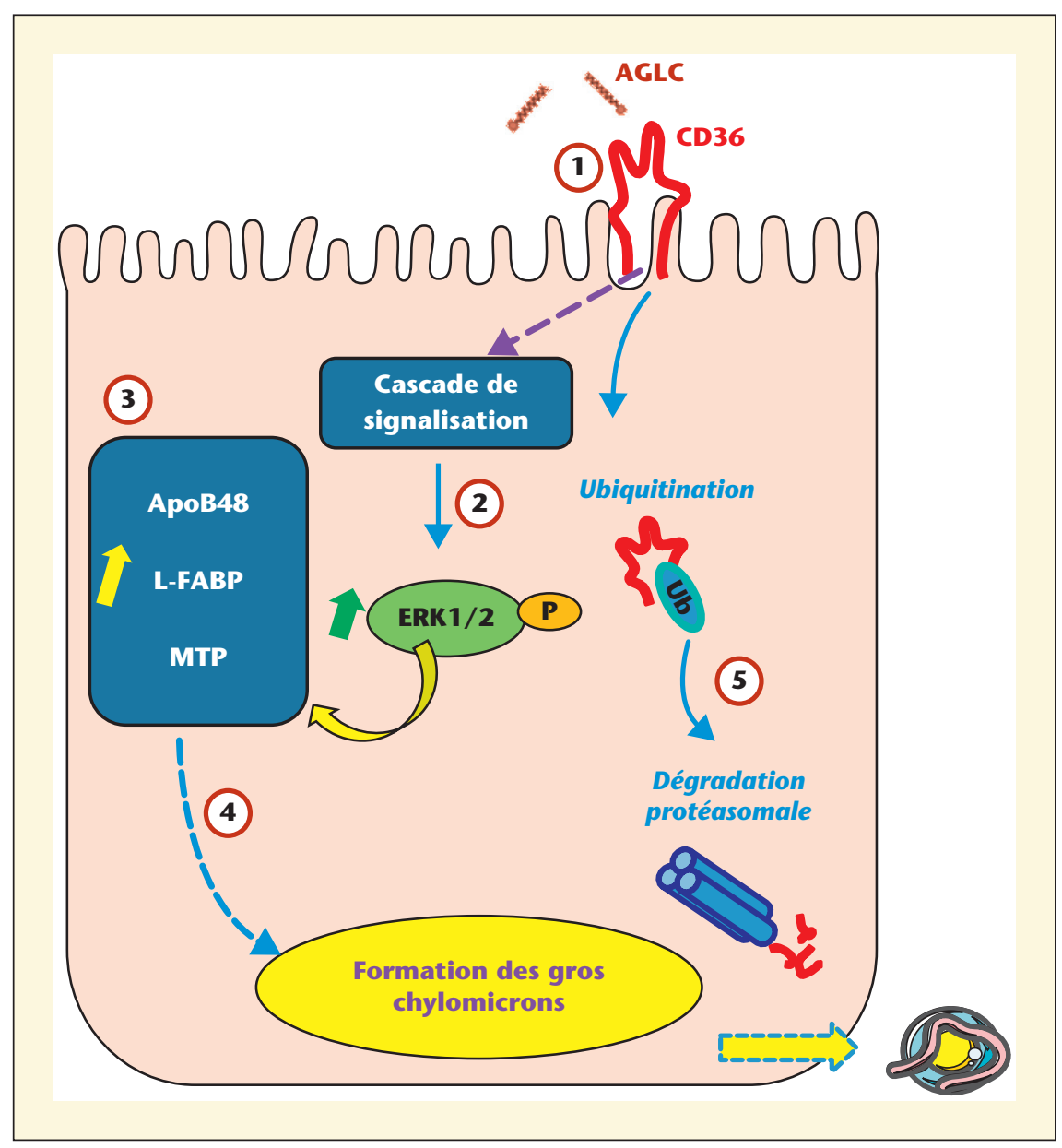

Figure 2. Rôle du CD36 dans l'absorption intestinale des lipides.1) Liaison des acides gras à longue chaîne (AGLC) issus de la digestion des triglycérides alimentaires avec le CD36. 2) Activation des ERK1/2. 3) et 4) Le CD36 via I'activation des ERK1/2 déclenche l'induction de I'ApoB48, de la MTP et de la L-FABP, trois protéines qui optimisent la synthèse des chylomicrons. 5) Les AGLC déclenchent une dégradation du CD36 par la voie ubiquitineprotéasome : désensibilisation. 
En conclusion, au début de la phase d'absorption intestinale, l'interaction entre CD36 et les AGLC provenant de I'hydrolyse des TG alimentaires déclencherait une activation du métabolisme intestinal conduisant à la formation de chylomicrons riches en TG donc de grande taille efficacement dégradés dans le sang (figure 2 ). Ce rôle de lipido-récepteur pour le CD36 intestinal expliquerait pourquoi les animaux et les patients déficients en CD36 sécrètent de petits chylomicrons à l'origine d'une hypertriglycéridémie postprandiale (Drover et al., 2005 ; Masuda et al., 2009). CD36 est donc la première étape d'un mécanisme qui conduit à une adaptation postprandiale du métabolisme entérocytaire des lipides permettant de prendre en charge de grandes quantités de lipides alimentaires.

\section{Conclusion et perspectives}

Cette revue montre que des avancées importantes ont été réalisées sur les différentes étapes de l'absorption des lipides par la découverte progressive des fonctions des différentes LBP. Cependant, le rôle physiologique des LBP membranaires est encore ambigu même si les fonctions émergentes démontrent que ce ne sont pas des transporteurs efficaces des AGLC en période d'absorption des lipides. La recherche du rôle physiologique des LBP membranaires au niveau intestinal nécessite la prise en compte de la spécificité intestinale (couche d'eau non agitée, micelles mixtes, présence de grandes quantités de lipides par intermittence, etc.).

Au niveau du mécanisme d'absorption, l'étape de lipidation de I'ApoB48 est la moins connue et pourtant elle est critique pour la formation de chylomicrons bien dégradés dans la circulation sanguine (taille).

Cette revue met en exergue que l'intestin est capable d'adapter ses capacités d'absorption et la qualité des lipoprotéines sécrétées (taille et ApoC) à la teneur en lipides du repas. Cette adaptation semble dépendante de la détection des produits de digestion des lipides alimentaires par l'intermédiaire du CD36. Ce système de sensing semble faire intervenir un autre récepteur de la même famille que CD36, le SRB1 (voir I'article présenté dans ce numéro par Beaslas et al., 2012). Ainsi une perturbation de ce système de sensing au niveau des récepteurs ou de leur signalisation est susceptible d'altérer les capacités d'absorption et la lipémie postprandiale.

Finalement, ce sensing entérocytaire peut affecter la biodisponibilité de tous les nutriments ou molécules lipophiles véhiculés par les chylomicrons (vitamines A, D, E, TG, cholestérol, médicaments). Ces dernières découvertes ouvrent de nouvelles perspectives thérapeutiques ou nutritionnelles pour moduler les capacités d'absorption et limiter l'hypertriglycéridémie postprandiale.

Financements : Agence Française de la Recherche : programme SENSOFAT.

\section{RÉFÉRENCES}

Atshaves BP, Foxworth WB, Frolov A, et al. Cellular differentiation and I-FABP protein expression modulate fatty acid uptake and diffusion. Am J Physiol 1998 ; 274 : C633-644.

Beaslas O, Cueille C, Delers F, Chateau D, Chambaz J, Rousset $M$, Carrière V. Détection luminale des micelles lipidiques. OCL 2012 ; 19. (Sous presse) doi : 10.1684/ocl.2012. 0448.

Berriot-Varoqueaux $\mathrm{N}$, Dannoura $\mathrm{AH}$, Moreau A, et al. Apolipoprotein B48 glycosylation in abetalipoproteinemia and Anderson's disease. Gastroenterology $2001 ; 121$ : 1101-8.

Bray GA, Paeratakul S, Popkin BM. Dietary fat and obesity: a review of animal, clinical and epidemiological studies. Physiol Behav 2004 ; $83: 549-55$.

Cartwright IJ, Higgins JA. Increased dietary triacylglycerol markedly enhances the ability of isolated rabbit enterocytes to secrete chylomicrons: an effect related to dietary fatty acid composition. J Lipid Res 1999 ; 40 : 1858-66.

Chow SL, Hollander D. Linoleic acid absorption in the unanesthetized rat: mechanism of transport and influence of luminal factors on absorption. Lipids $1979 ; 14$ : 378-85.

Corsico B, Cistola DP, Frieden C, Storch J. The helical domain of intestinal fatty acid binding protein is critical for collisional transfer of fatty acids to phospholipid membranes. Proc Natl Acad Sci U S A 1998 ; 95 : 12174-8.

de Wit NJ, Boekschoten MV, Bachmair EM, et al. Dose-dependent effects of dietary fat on development of obesity in relation to intestinal differential gene expression in C57BL/6] mice. PLoS One 2011 ; 6 : e19145.

Defoort C, Vincent-Baudry S, Lairon D. Effects of 3-month Mediterranean-type diet on postprandial TAG and apolipoprotein B48 in the Medi-RIVAGE cohort. Public Health Nutr $2011 ; 14: 2302-8$.

Doege $H$, Stahl A. Protein-mediated fatty acid uptake: novel insights from in vivo models. Physiology (Bethesda) 2006 ; 21 : 259-68.

Drover VA, Ajmal M, Nassir F, et al. CD36 deficiency impairs intestinal lipid secretion and clearance of chylomicrons from the blood. J Clin Invest 2005 ; 115 : 1290-7.

Drover VA, Nguyen DV, Bastie CC, et al. CD36 mediates both cellular uptake of very long chain fatty acids and their intestinal absorption in mice. J Biol Chem 2008 ; 283 : 13108-15.

El-Yassimi A, Hichami A, Besnard P, Khan NA. Linoleic acid induces calcium signaling, Src kinase phosphorylation, and neurotransmitter release in mouse CD36positive gustatory cells. I Biol Chem 2008 ; 283 : 12949-5.

Federico LM, Naples M, Taylor D, Adeli K. Intestinal insulin resistance and aberrant production of apolipoprotein B48 lipoproteins in an animal model of insulin resistance and metabolic dyslipidemia: evidence for activation of protein tyrosine phosphatase$1 \mathrm{~B}$, extracellular signal-related kinase, and sterol regulatory element-binding protein-1c in the fructose-fed hamster intestine. Diabetes 2006 ; 55 : 1316-26.

Frochot V, Alqub M, Cattin AL, et al. The transcription factor HNF-4alpha: a key factor of the intestinal uptake of fatty acids in mouse. Am J Physiol Gastrointest Liver Physiol 2012b ; 302 : G1253-63.

Garcia-Martinez C, Marotta M, MooreCarrasco R, et al. Impact on fatty acid metabolism and differential localization of FATP1 and FAT/CD36 proteins delivered in cultured human muscle cells. Am J Physiol Cell Physiol 2005 ; 288 : C1264-1272.

Gertow K, Bellanda M, Eriksson P, et al. Genetic and structural evaluation of fatty acid transport protein-4 in relation to markers of the insulin resistance syndrome. J Clin Endocrinol Metab 2004 ; 89 : 392-9.

Gimeno RE, Hirsch DJ, Punreddy S, et al. Targeted deletion of fatty acid transport protein-4 results in early embryonic lethality. J Biol Chem 2003 ; 278 : 49512-6.

Hall AM, Wiczer BM, Herrmann T, Stremmel W, Bernlohr DA. Enzymatic properties of purified murine fatty acid transport protein 4 and analysis of acyl-CoA synthetase activities in tissues from FATP4 null mice. / Biol Chem $2005 ; 280$ : 11948-54.

Holehouse EL, Liu ML, Aponte GW. Oleic acid distribution in small intestinal epithelial cells expressing intestinal-fatty acid binding protein. Biochim Biophys Acta $1998 ; 1390$ : 52-64. 
Ibrahimi A, Sfeir Z, Magharaie H, Amri EZ, Grimaldi P, Abumrad NA. Expression of the CD36 homolog (FAT) in fibroblast cells: effects on fatty acid transport. Proc Natl Acad Sci U S A 1996 ; 93 : 2646-51.

Iqbal J, Hussain MM. Intestinal lipid absorption. Am / Physiol Endocrinol Metab 2009 ; 296 : E1183-1194.

Jong MC, Hofker MH, Havekes LM. Role of ApoCs in lipoprotein metabolism: functional differences between $\mathrm{ApoC} 1, \mathrm{ApoC2}$, and ApoC3. Arterioscler Thromb Vasc Biol 1999 ; 19 : 472-84.

Karpe F, Olivecrona T, Hamsten A, Hultin M. Chylomicron/chylomicron remnant turnover in humans: evidence for margination of chylomicrons and poor conversion of larger to smaller chylomicron remnants. I Lipid Res 1997 ; 38 : 949-61.

Kondo $\mathrm{H}$, Minegishi $\mathrm{Y}$, Komine $\mathrm{YV}$, et al. Differential regulation of intestinal lipid metabolism-related genes in obesity-resistant $\mathrm{A} / \mathrm{J}$ vs obesity-prone C57BL/6] mice. Am J Physiol Endocrinol Metab 2006 ; 291 : E1092-1099.

Laugerette F, Passilly-Degrace P, Patris B, et al. CD36 involvement in orosensory detection of dietary lipids, spontaneous fat preference, and digestive secretions. / Clin Invest $2005 ; 115: 3177-84$

Lin MC, Arbeeny C, Bergquist K, Kienzle B, Gordon DA, Wetterau JR. Cloning and regulation of hamster microsomal triglyceride transfer protein. The regulation is independent from that of other hepatic and intestinal proteins which participate in the transport of fatty acids and triglycerides. J Biol Chem 1994 ; 269 : 29138-45.

Lobo MV, Huerta L, Ruiz-Velasco N, et al. Localization of the lipid receptors CD36 and CLA-1/SR-BI in the human gastrointestinal tract: towards the identification of receptors mediating the intestinal absorption of dietary lipids. J Histochem Cytochem 2001 ; $49: 1253-60$

Mansbach 2nd CM, Gorelick F. Development and physiological regulation of intestinal lipid absorption. II. Dietary lipid absorption, complex lipid synthesis, and the intracellular packaging and secretion of chylomicrons. Am J Physiol Gastrointest Liver Physiol 2007 ; 293 : G645-650.

Mariadason JM, Nicholas C, L'Italien KE, et al. Gene expression profiling of intestinal epithelial cell maturation along the cryptvillus axis. Gastroenterology $2005 ; 128$ : 1081-8.

Martin C, Chevrot M, Poirier H, PassillyDegrace P, Niot I, Besnard P. CD36 as a lipid sensor. Physiol Behav 2011 ; 105 : 36-42.

Martins IJ, Mortimer BC, Miller J, Redgrave TG. Effects of particle size and number on the plasma clearance of chylomicrons and remnants. J Lipid Res 1996 ; 37 : 2696-705.

Masuda D, Hirano K, Oku $\mathrm{H}$, et al. Chylomicron remnants are increased in the postprandial state in CD36 deficiency. / Lipid Res 2009 ; 50 : 999-1011.

Milger K, Herrmann T, Becker C, et al. Cellular uptake of fatty acids driven by the ER-localized acyl-CoA synthetase FATP4. / Cell Sci 2006 ; 119 : 4678-88.

Mora S, Rifai N, Buring JE, Ridker PM. Fasting compared with nonfasting lipids and apolipoproteins for predicting incident cardiovascular events. Circulation 2008 ; 118 : 993-1001.

Nassir F, Wilson B, Han X, Gross RW, Abumrad NA. CD36 is important for fatty acid and cholesterol uptake by the proximal but not distal intestine. I Biol Chem 2007 ; 282 : 19493-501.

Nauli AM, Nassir F, Zheng S, et al. CD36 is important for chylomicron formation and secretion and may mediate cholesterol uptake in the proximal intestine. Gastroenterology $2006 ; 131$ : 1197-207.

Neeli I, Siddiqi SA, Siddiqi S, et al. Liver fatty acid-binding protein initiates budding of prechylomicron transport vesicles from intestinal endoplasmic reticulum. I Biol Chem 2007 ; 282 : $17974-8$.

Newberry EP, Xie Y, Kennedy S, et al. Decreased hepatic triglyceride accumulation and altered fatty acid uptake in mice with deletion of the liver fatty acid-binding protein gene. J Biol Chem 2003 ; 278 : 51664 72.

Newberry EP, Xie Y, Kennedy SM, Luo J, Davidson NO. Protection against Western diet-induced obesity and hepatic steatosis in liver fatty acid-binding protein knockout mice. Hepatology 2006 ; 44 : 1191-205.

Niot I, Poirier H, Tran TT, Besnard P. Intestinal absorption of long-chain fatty acids: evidence and uncertainties. Prog Lipid Res 2009 ; 48 : 101-15.

Olivecrona G, Beisiegel U. Lipid binding of apolipoprotein $\mathrm{CII}$ is required for stimulation of lipoprotein lipase activity against apolipoprotein Cll-deficient chylomicrons. Arterioscler Thromb Vasc Biol 1997 ; 17 : 1545-9.

Petit V, Arnould L, Martin P, et al. Chronic high-fat diet affects intestinal fat absorption and postprandial triglyceride levels in the mouse. J Lipid Res 2007 ; 48 : 278-87.

Poirier H, Degrace P, Niot I, Bernard A, Besnard $\mathrm{P}$. Localization and regulation of the putative membrane fatty-acid transporter (FAT) in the small intestine. Comparison with fatty acid-binding proteins (FABP). Eur J Biochem 1996 ; 238 : 368-73.
Prows DR, Schroeder F. Metallothionein-IIA promoter induction alters rat intestinal fatty acid binding protein expression, fatty acid uptake, and lipid metabolism in transfected L-cells. Arch Biochem Biophys 1997 ; 340 : 135-43.

Ross AC. Overview of retinoid metabolism. J Nutr $1993 ; 123$ : 346-50.

Shiau YF, Fernandez P, Jackson MJ, McMonagle S. Mechanisms maintaining a low-pH microclimate in the intestine. $A m$ J Physiol 1985 ; 248 : G608-617.

Shim J, Moulson CL, Newberry EP, et al. Fatty acid transport protein 4 is dispensable for intestinal lipid absorption in mice. / Lipid Res $2009 ; 50: 491-500$.

Silverstein RL, Febbraio M. CD36, a scavenger receptor involved in immunity, metabolism, angiogenesis, and behavior. Sci Signal 2009 ; 2 : re3.

Stahl A, Gimeno RE, Tartaglia LA, Lodish HF. Fatty acid transport proteins: a current view of a growing family. Trends Endocrinol Metab $2001 ; 12: 266-73$.

Stahl A, Hirsch DI, Gimeno RE, et al. Identification of the major intestinal fatty acid transport protein. Mol Cell 1999 ; 4 : 299-308.

Steinert RE, Beglinger C. Nutrient sensing in the gut: interactions between chemosensory cells, visceral afferents and the secretion of satiation peptides. Physiol Behav 2011; 105 : 62-70.

Stremmel W. Uptake of fatty acids by jejunal mucosal cells is mediated by a fatty acid binding membrane protein. J Clin Invest $1988 ; 82: 2001-10$.

Stremmel W, Lotz G, Strohmeyer G, Berk PD. Identification, isolation, and partial characterization of a fatty acid binding protein from rat jejunal microvillous membranes. I Clin Invest 1985a ; $75:$ 1068-76.

Stremmel W, Strohmeyer G, Borchard F, Kochwa S, Berk PD. Isolation and partial characterization of a fatty acid-binding protein in rat liver plasma membranes. Proceedings of the National Academy of Sciences USA $1985 \mathrm{~b} ; 82: 4-8$.

Stump DD, Zhou SL, Berk PD. Comparison of plasma membrane FABP and mitochondrial isoform of aspartate aminotransferase from rat liver. Am J Physiol 1993 ; 265 : G894-902.

Su X, Abumrad NA. Cellular fatty acid uptake: a pathway under construction. Trends Endocrinol Metab 2009 ; 20 : 72-7.

Sukhotnik I, Gork AS, Chen M, Drongowski RA, Coran AG, Harmon CM. Effect of low fat diet on lipid absorption and fatty-acid transport following bowel resection. Pediatr Surg Int $2001 ; 17$ : 259-64.

Swift LL, Jovanovska A, Kakkad B, Ong DE. Microsomal triglyceride transfer protein 
expression in mouse intestine. Histochem Cell Biol 2005 ; 123 : 475-82.

Tomkin GH, Owens D. The chylomicron: relationship to atherosclerosis. Int / Vasc Med 2012 ; 2012 : 784536.

Tran $\mathrm{TT}$, Poirier $\mathrm{H}$, Clement $\mathrm{L}$, et al. Luminal lipid regulates CD36 levels and downstream signaling to stimulate chylomicron synthesis. J Biol Chem 2011 ; 286 : 25201-10.

Tran TTT, Poirier H, Clement L, et al. CD36 Displays Features of a Lipid-Sensor Involved in Chylomicron Processing in the Rodent Small Intestine. Atheroscler Suppl $2010 ; 11$ : 82-3.

Tsai J, Qiu W, Kohen-Avramoglu R, Adeli K. MEK-ERK inhibition corrects the defect in VLDL assembly in HepG2 cells: potential role of ERK in VLDL-ApoB100 particle assembly.
Arterioscler Thromb Vasc Biol 2007 ; 27 : 211-8.

Tso P, Liu M. Apolipoprotein A-IV, food intake, and obesity. Physiol Behav 2004 ; $83: 631-43$.

Uchida A, Whitsitt MC, Eustaquio T, et al. Reduced triglyceride secretion in response to an acute dietary fat challenge in obese compared to lean mice. Front Physiol 2012 ; $3: 26$.

Vassileva G, Huwyler L, Poirier K, Agellon LB, Toth MJ. The intestinal fatty acid binding protein is not essential for dietary fat absorption in mice. FASEB / $2000 ; 14$ : 2040-6.

Weinberg RB, Gallagher JW, Fabritius MA, Shelness GS. ApoA-IV modulates the secre- tory trafficking of $a p o B$ and the size of triglyceride-rich lipoproteins. / Lipid Res 2012; $53: 736-43$.

Xiang SQ, Cianflone K, Kalant D, Sniderman AD. Differential binding of triglyceride-rich lipoproteins to lipoprotein lipase. / Lipid Res 1999 ; 40 : 1655-63.

Xie Y, Luo J, Kennedy S, Davidson NO. Conditional intestinal lipotoxicity in Apobec1-/- Mttp-IKO mice: a survival advantage for mammalian intestinal apolipoprotein B mRNA editing. J Biol Chem 2007 ; 282 : 33043-51.

Xie Y, Newberry EP, Young SG, et al. Compensatory increase in hepatic lipogenesis in mice with conditional intestine-specific Mttp deficiency. J Biol Chem 2006 ; 281 : 4075-86. 